\title{
Tail-to-Head Tandem Duplication and Simple Repetitive Sequences of the Cytoplasmic Actin Genes in Greenling Hexagrammos otakii (Teleostei; Scorpaeniformes)
}

\author{
Sang Yoon Lee, Dong Soo Kim and Yoon Kwon Nam* \\ Department of Marine Bio-Materials and Aquaculture, Pukyong National University, Busan 608-737, Korea
}

\begin{abstract}
We characterized a cytoplasmic actin gene locus in greenling Hexagrammos otakii (Scorpaeniformes). Genomic clones isolated from the greenling DNA library contained two homologous cytoplasmic actin gene copies (HObact2.1 and HObact2.2) in a tail-tohead orientation. Their gene structure is characterized by six translated exons and one non-translated exon. Exon-intron organization and the nucleotide sequences of the two actin gene isoforms are very similar. However, only the HObact2.1 isoform contains microsatellite-like, dinucleotide repeats in the $5^{\prime}$-flanking region (named HOms2002) and intron 1 following the non-translated exon 1 (named HOms769). One microsatellite locus (HOms769) was highly polymorphic while the other (HOms2002) was not. Based on bioinformatic analysis, different transcription factor binding motifs are related to stress and immune responses in the two actin isoforms. Semiquantitative and real-time reverse transcription-PCR assays showed that both isoform transcripts were detectable ubiquitously in all the tissues examined. However, the basal expression levels of each isoform varied across tissues. Overall, the two isoforms showed a similar, but not identical, expression pattern. Our data suggest that the cytoplasmic actin genes may be the result of a recent duplication event in the greenling genome, which has not experienced significant subfunctionalization in their housekeeping roles.
\end{abstract}

Key words: Cytoplasmic actin, Gene duplication, SRS, Tissue distribution, Hexagrammos otakii

\section{Introduction}

Cytoskeletal actin provides a mechanical support for the cell and mediates cell motility and organelle movement, which are associated with several dynamic cellular activities such as morphological and developmental changes, macromolecule transport, and chemotaxis (Reisler and Egelman, 2007). Furthermore, actin is involved in signal transduction (Percipalle and Visa, 2006), RNA localization (Kustermans et al., 2008), apoptosis (Franklin-Tong and Gourlay, 2008), and nitric oxide (NO) regulation (Ji et al., 2007). In addition, the 5'-flanking region of cytoskeletal actin genes is capable of driving the expression of downstream structural genes when it is included in a chimeric transgene construct as a regulatory element (Nam et al., 2008).

In teleosts, genetic determinants of cytoskeletal actin have been isolated from various species belonging to a wide array of taxonomic positions. Although the coding regions of cytoskeletal actin are well characterized, their genomic organization and upstream regulatory regions require further investigation (see Venkatesh et al., 1996; Cho et al., 2011). Teleosts experienced whole-genome duplication in their evolutionary history and many teleost species may exhibit additional, locus-specific gene duplications in their genomes (Jaillon et al., 2004). Hence, the teleost group likely encodes diverse paralog isoforms in their genomes, and some of these paralogs may
Open Access http://dx.doi.org/10.5657/FAS.2011.0303

This is an Open Access article distributed under the terms of the Creative Commons Attribution Non-Commercial License (http://creativecommons. org/licenses/by-nc/3.0/) which permits unrestricted non-commercial use, distribution, and reproduction in any medium, provided the original work is properly cited.
Received 6 September 2011; Accepted 29 November 2011

*Corresponding Author

E-mail: yoonknam@pknu.ac.kr 
undergo subfunctionalization or neofunctionalization. In vertebrates, the actin multigene family evolved into six or more isoforms from a single ancestral form (Miwa et al., 1991; Venkatesh et al., 1996), and previous studies have suggested that a greater spectrum of actin isoforms may exist in the fish genome compared to representative mammalian species (Venkatesh et al., 1996). Duplicated (diverged) actin genes are known to exist in the teleost genomes, but their organization has not been extensively studied.

Greenling Hexagrammos otakii (Teleostei; Scorpaeniformes) is a commercially important marine food fish in Korea and China, and its market demand has been gradually increasing. This species is also a candidate for a marine launching program in Korea because of its nonmigratory and gathering behavior around artificial reefs. Although the culturing techniques and artificial manipulation required throughout its life cycle have not been established, various studies have been performed to better understand their physiology, with a focus on reproduction, growth, metabolism, and stress (Nam and Kim, 2002; Kang et al., 2004; Kim et al., 2009). Discovery and characterization of useful genetic markers and gene sequences are essential to gain a deeper insight into the physiology of a species. As a part of our long-term research to collect genomic and gene information from this species to aid its genetic breeding program, the objective of this study was to characterize the genomic organization and gene structure of the duplicated cytoplasmic actin isoforms.

\section{Materials and Methods}

\section{Fish specimens and nucleic acid libraries}

Greenling specimens were purchased from a local fish farm and transferred to a laboratory culture station. Genomic DNA samples were obtained from a fin clip or whole blood using the conventional SDS/proteinase K method followed by ethanol precipitation. To construct the greenling cDNA library, total RNA was extracted from liver and brain tissues using the RNeasy Midi Kit (Qiagen, Hilden, Germany). After purification of the poly(A)+ RNA fraction using the mRNA Isolation Kit (Promega, Madison, WI, USA), an aliquot ( $5 \mu \mathrm{g})$ of poly $(\mathrm{A})+\mathrm{RNA}$ was used as the template for cDNA synthesis. Construction of the cDNA libraries was performed using the Lambda Zap cDNA Synthesis Kit (Stratagene, La Jolla, CA, USA) according to the manufacturer's instruction. The genomic DNA library was constructed using whole blood DNA purified from a male adult using the Lambda Gem 11 Cloning System (Promega) according to manufacturers' protocol.

\section{Isolation of genomic genes using DNA library screening}

Based on our preliminary expressed sequence tag (EST) analysis of greenling brain and liver cDNA libraries (unpublished data), an EST clone with significant homology to vertebrate cytoplasmic actin was identified and used as a probe to perform a series of filter screenings of the greenling genomic DNA library. The EST clone $(0.6 \mathrm{~kb})$ was PCR-labeled with digoxygenin-11-dUTP (Roche Applied Science, Manheim, Germany). Approximately $5 \times 10^{5}$ phage plaques were screened by plaque hybridization using the DIG DNA Labeling and Detection Kit (Roche Applied Science). The selected phages were amplified using KW251 Escherichia coli strain and the phage DNA was purified using the Wizard Lambda Phage Prep Kit (Promega). Purified phage DNA was digested with $\mathrm{SacI}$ and subcloned into a pBluescript KS II phagemid vector (Stratagene). The sequence of the DNA inserts in each subclone was determined using the transposon-mediated shotgun sequencing method (EZ-Tn5 KAN-2 Kit; Epicentre, Madison, WI, USA) according to the manufacturer's instructions. Based on the contig assembly using Sequencher (Gene Codes, Ann Arbor, MI, USA), overlapping genomic clones were PCR-isolated and sequenced to validate the representative sequence of the cytoplasmic actin gene.

\section{Isolation of cDNA sequences}

Based on genomic sequence analysis, type-specific reverse transcription (RT)-PCR primer pairs (HOact2.1c-1F/1R and HOact2.2c-1F/1R) were designed to amplify the complete open reading frame (ORF) of each isoform. Total RNA (500 ng) from the brain or gill was used as a template for RT-PCR isolation. Oligonucleotide primers and thermal cycling conditions used in this study are summarized to Table 1 . The amplification product was sequenced after TA cloning into the pGEM-T Easy Vector (Promega). Based on the ORF-containing sequences, the $5^{\prime}$ - and 3 '-end untranslated region (UTR) sequences of each isoform were obtained by vectorette PCR, which was performed using template DNA prepared from greenling brain or liver cDNA libraries (excised stocks) and two vector primers (SK and T7). Binding sites are located at either end of the multiple cloning sites in the phagemid vector (pBluescript SK; Stratagene) used for construction of the cDNA libraries. Isoform-specific primers included in the vectorette PCR as either forward or reverse primers paired with SK or T7 vector primers were HOact2.1-vec1 and HOact2.2vec1, respectively. Based on the contig assembly of ORF and ends sequences, we determined the full-length cDNA sequence of each isoform.

\section{Bioinformatic sequence analysis}

Using NCBI GenBank BLAST, we searched for orthologs of the full-length cDNA sequences. Putative ORFs of each isoform were identified using ORF Finder (http://www.ncbi. nlm.nih.gov/gorf/gorf.html). Predicted molecular mass and theoretical pI values were calculated using the ProtParam 
tool (http://www.expasy.org/tools/protparam.html). Multiple sequence alignments were performed using a ClustalW algorithm (http:/www.genome.jp/tools/clustalw/). Genomic structure and organization were compared using representative orthologs and/or paralogs obtained from the public databases, including GenBank and Ensembl (http://www.ensembl. org/). Putative transcription factor (TF)-binding motifs in the 5 -flanking upstream region of the actin isoforms were identified using TFSEARCH (http://www.cbrc.jp/research/db/ TFSEARCH.html) and Transcription Element Search System (TESS; http://www.cbil.upenn.edu/cgi-bin/tess/tess). Identification of repetitive sequences was performed using the Unipro UGENE (http://ugene.unipro.ru/).

\section{Typing of simple sequence repeats (SSRs)}

We identified microsatellite-like, SSRs in the noncoding region of one actin isoform. To identify polymorphism among greenling individuals, two PCR primer pairs (HOms769 1F/1R and HOms2002 1F/1R) were designed to amplify each of the two microsatellite regions. In total, 48 individuals randomly obtained from three different domestic markets were subjected to microsatellite typing. Genomic DNA was extracted from a caudal fin clip (as described above) and $200 \mathrm{ng}$ of purified DNA was used as a template for PCR amplification. The PCR product $(5 \mu \mathrm{L})$ was separated on $1.5 \%$ agarose gel and visualized by ethidium bromide staining to examine any polymorphism in PCR product length among individuals.

\section{Tissue distribution and basal expression of actin transcripts using a RT-PCR assay}

We performed real-time RT-PCR to examine the tissue distribution and basal expression of each actin isoform transcript. Total RNA was extracted from ten somatic tissues including the brain, eye, fin, gill, heart, intestine, kidney, liver, muscle, and spleen, which were obtained from 12 juvenile individuals (average body weight, $456 \pm 52.0 \mathrm{~g}$ ), as described above. Total RNA $(4 \mu \mathrm{g})$ was reverse-transcribed $\left(37^{\circ} \mathrm{C}\right.$ for $\left.60 \mathrm{~min}\right)$ into cDNA in a reaction volume of $40 \mu \mathrm{L}$ using the Omniscript Reverse Transcription Kit (Qiagen) according to the manufacturer's instructions. A conserved reverse primer for teleost $18 \mathrm{~S}$ rRNA (Fi18SrRNA-1R primer) was also included in the RT reaction at a $0.1 \mu \mathrm{M}$ final concentration to prepare the normalization standard across tissues. The RT product (cDNA) was diluted fourfold (for actins) or 40-fold (for 18S rRNA) with sterile distilled water, and $2 \mu \mathrm{L}$ of the diluted cDNA was used as a template for PCR amplification. Based on the preliminary optimization of thermal cycling conditions and PCR efficiency ( $E=10^{-1 / \text { slop }}$ - 1 in the standard curve for each gene), each actin isoform and $18 \mathrm{~S}$ rRNA control were amplified using the primers qHOact2.1-1F/1R (242 bp), qHOact2.2-1F/1R (218 bp), and q18S-1F/1R (261 bp), respectively. The reaction was performed on the iCycler iQ Real-Time Detection System

Table 1. Oligonucleotide primers used in this study

\begin{tabular}{lll}
\hline Primer & \multicolumn{1}{c}{ Sequence (5' to 3') } & \multicolumn{1}{c}{ Thermal cycling conditions } \\
\hline HOact2.1c-1F & CGCAGTCACAAACAAGAACCCTCC & 30 cycles at $94^{\circ} \mathrm{C}$ for $30 \mathrm{~s}, 58^{\circ} \mathrm{C}$ for $30 \mathrm{~s}$ and \\
HOact2.1c-1R & CTGGGCACAATCCTTCTCACACCC & $72^{\circ} \mathrm{C}$ for 1 min followed by a final elongation at \\
& & $72^{\circ} \mathrm{C}$ for 5 min
\end{tabular}

HOact2.2c-1F AGCAGTCACAGCCGAGAACCAGCT

HOact2.2c-1R CTGGGCACAATCCTTCTCACATCA

HOact2.1-vec1 ACTTGTGTATGCTGAGCCCGCAA

HOact2.2-vec1 TGGTGTATGCTGAGCTTGCGATG

$\begin{array}{ll}\text { HOms769 1F } & \text { AGCGTTATCTGCATGAGCCA } \\ \text { HOms769 1R } & \text { TGCAAACATTTCTGCACACCT } \\ \text { HOms2002 1F } & \text { CAGCCTCCTGAGTGTTTGCT } \\ \text { HOms2002 1R } & \text { TCCCTGACATCCATGTTGAG } \\ \text { Fi18SrRNA-1R } & \text { CAAGAATTTCACCTCTAGCGGC } \\ & \\ \text { qHOact2.1-1F } & \text { TGTGTATGCTGAGCCCGCAAC } \\ \text { qHOact2.1-1R } & \text { TACAGGTCTGTTGGGCAGTG } \\ \text { qHOact2.2-1F } & \text { GGTGTATGCTGAGCTTGCGAT } \\ \text { qHOact2.2-1R } & \text { ACTACAGGTCTGTTGGGCAT } \\ \text { q18S-1F } & \text { GCGGTAATTCCAGCTCCAAT } \\ \text { q18S-1R } & \text { AGAACCGGAGTCCTATTCCA }\end{array}$

30 cycles at $94^{\circ} \mathrm{C}$ for $30 \mathrm{~s}, 58^{\circ} \mathrm{C}$ for $30 \mathrm{~s}$ and $72^{\circ} \mathrm{C}$ for 30 s followed by a final elongation at $72^{\circ} \mathrm{C}$ for $5 \mathrm{~min}$
32 cycles at $94^{\circ} \mathrm{C}$ for $30 \mathrm{~s}, 56^{\circ} \mathrm{C}$ for $30 \mathrm{~s}$ and $72^{\circ} \mathrm{C}$ for $45 \mathrm{~s}$
32 cycles at $94^{\circ} \mathrm{C}$ for $45 \mathrm{~s}, 56^{\circ} \mathrm{C}$ for $30 \mathrm{~s}$ and $72^{\circ} \mathrm{C}$ for $45 \mathrm{~s}$

26 (end-point PCR) or 45 (real-time PCR) cycles at $94^{\circ} \mathrm{C}$ for $20 \mathrm{~s}, 58^{\circ} \mathrm{C}$ for $20 \mathrm{~s}$ and $72^{\circ} \mathrm{C}$ for $20 \mathrm{~s}$

27 (end-point PCR) or 45 (real-time PCR) cycles at $94^{\circ} \mathrm{C}$ for $20 \mathrm{~s}, 58^{\circ} \mathrm{C}$ for $20 \mathrm{~s}$ and $72^{\circ} \mathrm{C}$ for $20 \mathrm{~s}$

20 (end-point PCR) or 45 (real-time PCR) cycles at $94^{\circ} \mathrm{C}$ for $20 \mathrm{~s}, 58^{\circ} \mathrm{C}$ for $15 \mathrm{~s}$ and $72^{\circ} \mathrm{C}$ for $20 \mathrm{~s}$
Vectorette PCR for isolating 5 '- and 3 '-ends of HObact 2.1 and HObact 2.2 cDNAs

Amplification of microsatellite locus HOms 769

Amplification of microsatellite locus HOms2002

Preparation of normalized control (18S rRNA)

RT-PCR assay of HObact2.1 transcripts

RT-PCR assay of HObact 2.2 transcripts

RT-PCR assay of $18 \mathrm{~S}$ rRNA (normalization control)

Each PCR amplification reaction was performed with an initial denaturation step at $94^{\circ} \mathrm{C}$ for $2 \mathrm{~min}$.

RT-PCR, reverse transcription PCR. 


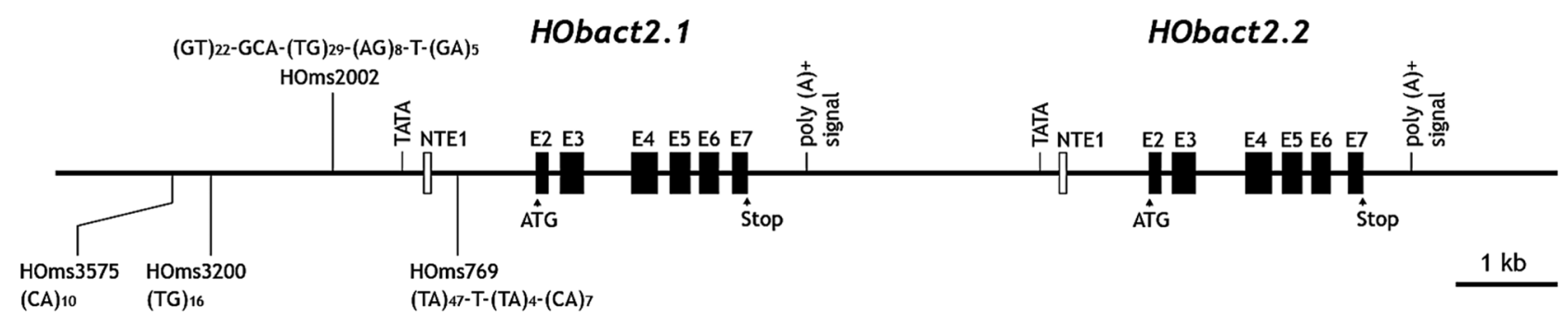

Fig. 1. Gene structure and genomic organization of two tandemly duplicated cytoplasmic actin genes (HObact2.1 and HObact2.2) in the Hexagrammos otakii genome. Coding exons (E2-E7) are indicated by closed, vertical boxes, while a non-translated exon 1 (NTE1) by open, vertical boxes. Translation start site (ATG codon) and stop codon of each actin isoform are noted by arrow heads. Putative TATA box and polyadenylation signal are also shown. The dinucleotide, microsatellite-like sequences found in HObact2.1 promoter and intron 1 are indicated along with their core sequences.

(Bio-Rad, Hercules, CA, USA) using the $2 \times$ iQ SYBR Green Supermix (Bio-Rad) with a reaction volume of $25 \mu \mathrm{L}$. Based on the amplification assays (performed in triplicate), the relative transcript levels of each actin isoform across tissues were normalized against the levels of the $18 \mathrm{~S}$ rRNA control using the following formula: relative expression $=\left[\left(1+E_{18 \mathrm{rRNA}}\right)\right.$ C18S rRNA $]^{-1} /\left[\left(1+E_{\text {ACTIN }}\right)^{\text {CtACTIN }}\right]^{-1}$, in which $E$ is the PCR efficiency and $\mathrm{Ct}$ is the threshold cycle number (Kubista et al., 2006; Schmittgen and Livak, 2008). Differences in the relative expression levels of each actin transcript among tissues were assessed by analysis of variance (ANOVA) followed by Duncan's multiple range test at $P=0.05$. The "endpoint" semiquantitative RT-PCR was conducted with the same primer pairs, and the amplification products were visualized on a $1.5 \%$ agarose gel using ethidium bromide staining.

\section{Results and Discussion}

\section{Gene structure, genomic organization, and cDNA sequences of greenling cytoplasmic actins}

Based on the sequence analysis of genomic DNA library clones followed by PCR isolation, the 14,813-bp genomic region containing two cytoplasmic actin genes was read at least twice in both directions. In the genomic region, two copies of the cytoplasmic actin gene existed tandemly in a tail-to-head organization. These two actin copies were named HOactb2.1 and HOactb2.2 (Fig. 1). Based on sequence comparison with its corresponding cDNA version (see below), HOactb2.1 has six translated exons (exons 2-7; 126, 240, 247, 192, 182, 144 bp including the stop codon) and one non-translated exon 1 (NET1; 69 bp). These seven exons are interrupted by six introns $(1026,104,507,109,95,181 \mathrm{bp}$, respectively, for introns 1-6). HOactb2.2 is also composed of seven exons (six translated exons and one NTE1; $66 \mathrm{bp}$ ) with a similar exon/ intron organization. The length of each translated HOactb2.2 exon is identical to that from HOactb2.1. Furthermore, the intron lengths of the second isoform $(797,102,509,109,95$, and 180, respectively, for introns 1-6) are also similar with those from HOactb2.1, except intron 1 following NTE1. The putative polyadenylation signal (AATAAA) is predicted at 503 and $477 \mathrm{bp}$ after the stop codon, respectively, in HOactb2.1 and HOactb2.2. The distance between the two actin gene copies (estimated based on the distance from the HOactb2.1 polyadenylation signal to the HOactb2.2 TATA box) is only 2.3 $\mathrm{kb}$. The full-length cDNA sequence of HOactb2.1 obtained by RT-PCR isolation and vectorette consists of $72 \mathrm{bp}$ of 5'UTR, 1,125 bp of a single ORF encoding a polypeptide of 375 amino acids (aa), and $552 \mathrm{bp}$ of a $3^{\prime}$-UTR including the stop codon and $21 \mathrm{bp}$ poly(A)+ tail. HOactb2.2 also encodes a 375-aa polypeptide with a homogenous cDNA structure composed of a 69-bp 5'-UTR, 1,125-bp ORF, and 525-bp 3'-UTR. Putative polyadenylation signals (AATAAA; also detected in the genomic sequence) are present 26 bp before the poly(A)+ tail in both isoforms. At the nucleotide level, the two isoforms share $99.3 \%$ sequence identity in the coding region with only 8 nucleotide substitutions. The noncoding regions (5'- and 3'UTRs) have more nucleotide differences. When comparing the 69-bp 5'-UTR in HOactb2.1 and HOactb2.2, nucleotide substitutions occur at 10 positions ( $85.5 \%$ sequence identity), while the 3 '-UTR has nucleotide differences at 46 positions (19 substitutions and 27 deletions) in the 531-bp aligned region (91.3\% identity). At the amino acid level, the two isoforms exhibit only one amino acid substitution $\left(\mathrm{Thr}^{323} \leftrightarrow \operatorname{Ser}^{323}\right)$ in the 375 -aa polypeptide (99.7\% identity). Consequently, they have similar protein characteristics including molecular weight (41.8 kDa) and theoretical pI value (5.3). The amino acid sequences of both isoforms share considerable homology to previously known vertebrate orthologs (data not shown).

All actin isoforms have remarkably similar amino acid sequences and polypeptide lengths across diverse metazoan organisms, demonstrating their high evolutionary constraints (Reece et al., 1992). However, compared to mammals, the teleostean group possesses a wider spectrum of actin isoforms, in which several isoforms show no clear orthology to known mammalian isoforms (Venkatesh et al., 1996; Kim et al., 2008). For example, of the various cytoplasmic actin isoforms identified in teleosts, the major $\beta$-cytoplasmic- 1 genes are clearly homologous to the mammalian isoforms (actbl and/or 


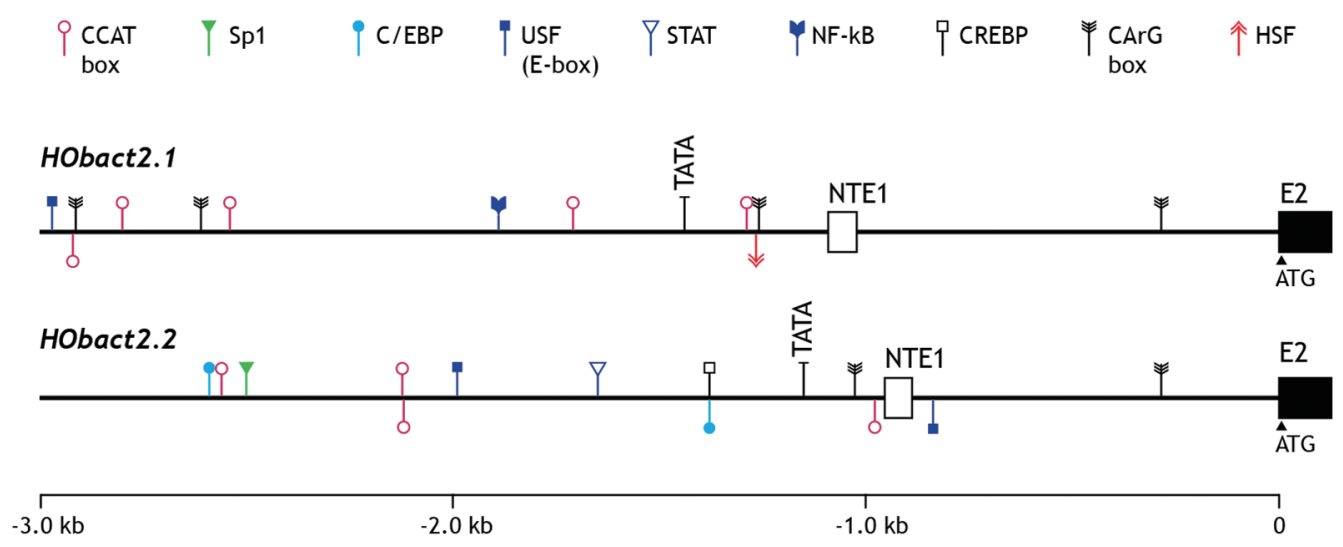

Fig. 2. Distribution patterns of potential transcription factor (TF) binding motifs in upstream regions of Hexagrammos otakii actin genes (HObact2.1 and HObact2.2). For each isoform copy, $-3.0 \mathrm{~kb}$ region from the ATG codon in the exon 2 (E2) was bioinformatically analyzed to identify putative TF binding motifs. Non-translated exon 1 (NTE1) indicates the non-translated exon 1; Sp1, stimulating protein; C/EBP, CAAT enhancer-binding protein; USF, upstream stimulatory factor; STAT, signal transducers and activator of transcription; NF-KB, nuclear factor kappa B; CREBP, cAMP-response element-binding protein; HSF, heat shock factor.

$\operatorname{actg} 1)$. Other isoforms are distinct from the mammalian counterparts, suggesting that the teleost group actin multigenes experienced different evolutionarily pathway(s) from that of mammals (Kim et al., 2008). In this study, the two greenling actin genes belong to the teleost minor cytoplasmic actin group (actb2) based on multiple sequence alignments and molecular phylogeny studies (data not shown). The number and position of the HOactb2.1 and HOactb2.2 introns exactly correspond to Takifugu rubripes $\beta$-cytoplasmic actin-2, characterized by Venkatesh et al. (1996). Furthermore, the similarity in coding nucleotide sequences, intron lengths, and head-to-tail arrangement between HOactb2.1 and HOactb2.2 unambiguously demonstrates that the two isoforms evolved by a recent gene duplication event. Our bioinformatic analyses also indicated that similar locus-specific duplication of actin gene copies occurred in the stickleback (Gasterosteus aculeatus; Gasterosteiformes) Ensembl scaffold (ENSGACG00000001330 and ENSGACG00000001357 in scaffold_225) and in zebrafish (Danio rerio; Cypriniformes) chromosome 25 (ENSDARG00000070646 and ENSDARG00000070647).

\section{Characteristics of the $5^{\prime}$-upstream region}

The 5'-flanking upstream regions (3 $\mathrm{kb}$ upstream from the translation start codon ATG) of HOactb2.1 and HOactb2.2 were analyzed to identify potential TF-binding motifs (Fig. 2). Each isoform contained binding motifs for various TFs in both the 5 '-flanking region and intron 1 following the nontranslated exon. In the proximal promoter region, HOactb2.1 and HOactb2.2 possess a conserved TATA box at $-1,348$ and $-1,065$ bp from the ATG site (i.e., -250 and -199 bp from the transcription start site; TSS), respectively. In both isoforms, one CAAT (CCAAT) and CArG [CC(A/T) $\left.{ }_{6} \mathrm{GG}\right]$ box are predicted between the TSS and TATA box, although the relative order and distance between these boxes are not identical between HOactb2.1 and HOactb2.2. These essential boxes are considered key elements to allow for constitutive and ubiquitous expression of the cytoplasmic actin genes in vertebrate tissues (Reece et al., 1992; Kim et al., 2008). The two isoforms exhibit four (HOactb2.1) and three (HOactb2.2) additional copies of CAAT boxes in their upstream regions. Fairly consistent with other beta-cytoskeletal teleost actin genes, $\mathrm{HO}$ actb2.1 and HOactb2.2 have one CArG box (a trans-acting factor-binding site) at a conserved position in intron 1, which is thought to function as an enhancer in the regulation of the cytoplasmic actin (Liu et al., 1991; Noh et al., 2003). Additionally, HOactb2.1 (but not HOactb2.2) has two more CArG boxes in its distal promoter region. Besides these known TF binding sites, both HOactb2.1 and HOactb2.2 likely possess motifs associated with stress and immune responses. They include binding sites for upstream stimulatory factor (USF; CANNTG), CAAT enhancer-binding protein (C/EBP; TTDNGNAA), stimulating protein (Sp1; GGCRGGG), heat shock factor (HSF; RGAANRTTC), nuclear factor kappa B (NF- $\kappa$; GGGRNNYYCC), signal transducers and activator of transcription (STAT; TTCNNNGAA), and cAMP-response element-binding protein (CREBP; TGACGY). Although these TFs are known to regulate immune- and stress-responsive genes (Anderson, 2000; Truksa et al., 2007; Cho et al., 2009), whether they modulate HOactb2.1 and HOactb2.2 regulation is not clear. Thus, examination of their gene expression under various stimulatory challenges is required to evaluate the involvement of these bioinformatically identified TFs in the transcription of $H$. otakii actin genes. Although the regulatory machinery (including these responsive TFs) has not been empirically characterized in fish cytoplasmic actins, our findings are similar to previous bioinformatic observations of other fish cytoskeletal actin genes that predicted different stress-respon- 


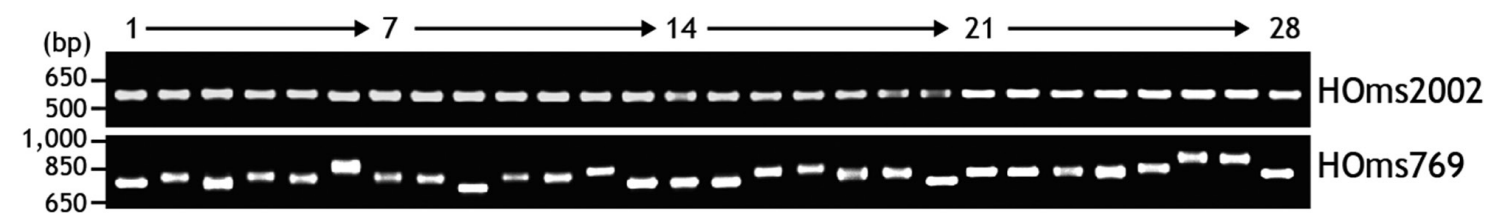

Fig. 3. Representative ethidium bromide-stained agarose gel showing the PCR products amplified from either two selected microsatellite loci (HOms769 in intron 1 or HOms2002 in the promoter region) found in the HObact 2.1 isoform. Only the results from 28 individuals are shown out of 48 individuals were assessed. The numbers on the top indicate the fish identification number.

sive TF-binding motifs in their regulatory regions (Lee et al., 2009; Cho et al., 2011). In addition, our data are partially supported by the fact that mRNA expression of cytoskeletal actin genes is modulated depending on the developmental stage, stress state, or pathological condition of the mammal. Thus, caution is required when using actin gene expression as an invariant, internal control for gene expression assays (Chaponnier and Gabbiani, 2004; Filby and Tyler, 2007; Small et al., 2008).

\section{Simple sequence repeats}

Of the two $H$. otakii actin isoforms, HOactb2.1 contained SSRs in the 5'-flanking region and intron 1. The HOactb2.1 upstream region contained four microsatellite loci (three in the promoter region and one in intron 1). All SSRs found in HOactb2.1 were dinucleotide repeats: $\left[(\mathrm{TA})_{47}-\mathrm{T}-(\mathrm{TA})_{4}-(\mathrm{CA})_{7}\right.$ at position -769 to $-653 \mathrm{bp}$ from the ATG start codon], [(GT) ${ }_{22}{ }^{-}$ GCA-(TG) $)_{29}-(\mathrm{AG})_{8}-\mathrm{T}-(\mathrm{GA})_{5}$ at $-2,002$ to $\left.-1,871 \mathrm{bp}\right],\left[(\mathrm{TG})_{16}\right.$ at $-3,200$ to $-3,169 \mathrm{bp}$ ] and $\left[(\mathrm{CA})_{10}\right.$ at $-3,575$ to $-3,556 \mathrm{bp}$ ] (see also Fig. 1). As a preliminary experiment, we selected two longer SSR regions located at positions -769 (HOms 769) and -2,002 (HOms2002), and evaluated whether they were polymorphic among individuals. Using the primer pairs HOms769 1F/1R (for HOms769 locus) and HOms2002 1F/1R (for HOms2002 locus), the two microsatellite loci were independently amplified using PCR. As shown in Fig. 3, the SSR in intron 1 (HOms 769) was highly polymorphic, even based on the conventional electrophoretic separation of the amplification products, whereas the SSR in the promoter region (HOms2002) was much less variable among individuals. Microsatellite SSR markers are useful to assess genetic diversity and develop molecular breeding techniques in fish due to their high level of polymorphism and codominant inheritance (Liu and Cordes, 2004). Microsatellite loci have also been isolated from a dinucleotide-enriched greenling genomic library (Chen et al., 2009). The HOms 769 locus identified in this study could be added to a polymorphic microsatellite marker set to address the genetic diversity of the species. Evaluating its potential use for cross-species amplification in the closely related Hexagrammos species would also be valuable. However, the possible number of alleles for this locus in different $H$. otakii populations should be characterized using genotyping analyses.

\section{Tissue distribution and basal expression levels}

Based on the real-time RT-PCR assay, each greenling actin isoform was detected ubiquitously in all the tissues examined, although the basal expression levels varied among tissues (Fig. 4). HOactb2.1 transcripts were actively expressed in the intestine, kidney, and gills, while its expression level was lowest in the liver and skeletal muscle $(P<0.05)$. Other tissues, includ-
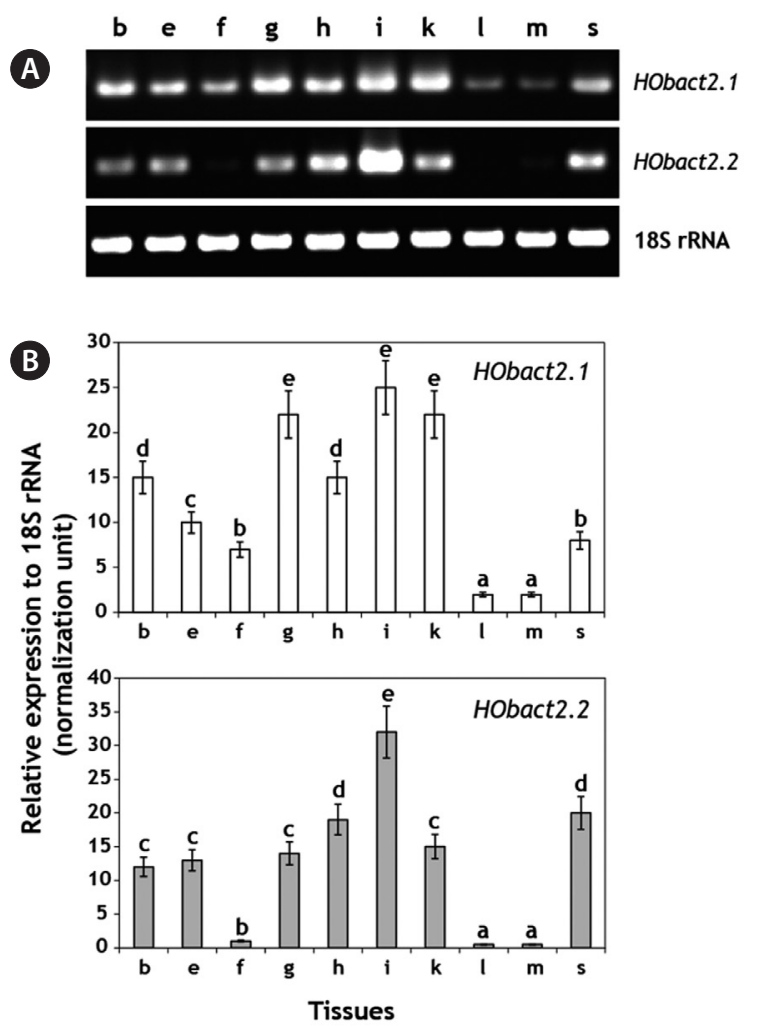

Fig. 4. Tissue distributions and basal expression levels of the two Hexagrammos otakii actin gene (HObact2.1 and HObact2.2) transcripts, as assessed by semi-quantitative reverse transcription (RT)-PCR (A) and realtime RT-PCR (B) based on the normalization against 18S rRNA control. Tissue abbreviations are brain (b), eye (e), fin ( $f$ ), gill (g), heart (h), intestine (i), kidney (k), liver (l), skeletal muscle $(\mathrm{m})$ and spleen $(\mathrm{s})$. In real-time RT-PCR analysis, histograms with the same letters are not significantly different $(P>0.05)$ based on the ANOVA followed by Duncan's multiple range tests. 
ing the brain, eye, fin, heart, and spleen, showed a moderate level of HOactb2.1 mRNA expression. HOactb2.2 transcripts showed a more intestine-enriched distribution $(P<0.05)$ than HOactb2.1. Overall, the two isoforms had a similar, but not identical, pattern of tissue expression with high levels in the intestine and low levels in the liver and skeletal muscle. The ubiquitous distribution pattern of $H$. otakii actin transcripts is not unexpected considering its essential housekeeping roles in most cell types. Furthermore, their overall pattern (high expression in the intestine and kidney and low expression in the skeletal muscle and liver) agrees with the expression of cytoplasmic actin genes from other teleost species (Kim et al., 2008; Lee et al., 2009). Meanwhile, Venkatesh et al. (1996) suggested that actin gene copies that experienced significant subfunctionalization after gene duplication would have different tissue distribution patterns, as exemplified by diverse actin paralogs in the pufferfish genome. However, based on the similar tissue expression patterns of HOactb2.1 and $\mathrm{HO}$ actb2.2, the two greenling actin copies (which recently duplicated) have not significantly differentiated in their basic functions. Nevertheless, further study to evaluate any differential expression in response to various experimental challenges are required since the two $H$. otakii actin isoforms have different TF-binding motifs in their regulatory regions.

In summary, two tandemly existing cytoplasmic actin genes were isolated and characterized from a greenling (H. otakii). They have a similar genomic organization and gene structure with a high degree of sequence identity at both the nucleotide and amino acid levels. However, in spite of their similar gene structures, the distribution pattern of TF-binding motifs in the regulatory region was different between the two isoform copies. Only one isoform (HObact2.1) had multiple SSRs in either the 5'-flanking region or intron 1. One of these SSRs is highly polymorphic among individuals. The mRNA tissue distribution pattern of the two actin isoforms was very similar, which agrees with vertebrate cytoskeletal and non-muscle actins. Collectively, the two actin isoforms in the H. otakii genome evolved from a recent gene duplication event and have not yet experienced significant subfunctionalization in their essential housekeeping roles.

\section{Acknowledgments}

This study was supported by a research fund from the Ministry of Land, Transport and Maritime Affairs, Republic of Korea (Project \#20088033-1).

\section{References}

Anderson KV. 2000. Toll signaling pathways in the innate immune response. Curr Opin Immunol 12, 13-19.

Chaponnier C and Gabbiani G. 2004. Pathological situations character- ized by altered actin isoform expression. J Pathol 204, 386-395.

Chen SL, Miao GD, Shao CW, Tian YS and Liao X. 2009. Isolation and characterization of polymorphic microsatellite loci from fat greenling (Hexagrammos otakii). Conserv Genet 10, 1429-1431.

Cho YS, Lee SY, Kim KH, Kim SK, Kim DS and Nam YK 2009. Gene structure and differential modulation of multiple rockbream (Oplegnathus fasciatus) hepcidin isoforms resulting from different biological stimulations. Dev Comp Immunol 33, 46-58.

Cho YS, Lee SY, Kim YK, Kim DS and Nam YK. 2011. Functional ability of cytoskeletal $\beta$-actin regulator to drive constitutive and ubiquitous expression of a fluorescent reporter throughout the life cycle of transgenic marine medaka Oryzias dancena. Transgenic Res 20, 1333-1355.

Filby AL and Tyler CR. 2007. Appropriate 'housekeeping' genes for use in expression profiling the effects of environmental estrogens in fish. BMC Mol Biol 8, 10.

Franklin-Tong VE and Gourlay CW. 2008. A role for actin in regulating apoptosis/programmed cell death: evidence spanning yeast, plants and animals. Biochem J 413, 389-404.

Jaillon O, Aury JM, Brunet F, Petit JL, Stange-Thomann N, Mauceli E, Bouneau L, Fischer C, Ozouf-Costaz C, Bernot A, Nicaud S, Jaffe D, Fisher S, Lutfalla G, Dossat C, Segurens B, Dasilva C, Salanoubat M, Levy M, Boudet N, Castellano S, Anthouard V, Jubin C, Castelli V, Katinka M, Vacherie B, Biémont C, Skalli Z, Cattolico L, Poulain J, De Berardinis V, Cruaud C, Duprat S, Brottier P, Coutanceau JP, Gouzy J, Parra G, Lardier G, Chapple C, McKernan KJ, McEwan P, Bosak S, Kellis M, Volff JN, Guigó R, Zody MC, Mesirov J, Lindblad-Toh K, Birren B, Nusbaum C, Kahn D, Robinson-Rechavi M, Laudet V, Schachter V, Quétier F, Saurin W, Scarpelli C, Wincker P, Lander ES, Weissenbach J, Roest Crollius H. 2004. Genome duplication in the teleost fish Tetraodon nigroviridis reveals the early vertebrate proto-karyotype. Nature 431, 946-957.

Ji Y, Ferracci G, Warley A, Ward M, Leung KY, Samsuddin S, Lévêque C, Queen L, Reebye V, Pal P, Gkaliagkousi E, Seager M and Ferro A. 2007. $\beta$-Actin regulates platelet nitric oxide synthase 3 activity through interaction with heat shock protein 90. Proc Natl Acad Sci U S A 104, 8839-8844.

Kang HW, Chung EY and Kim JH. 2004. Sexual maturation and spawning characteristics in greenling Hexagrammos otakii of the west coast in Korea. J Aquac 17, 30-38.

Kim KY, Lee SY, Cho YS, Bang IC, Kim DS and Nam YK. 2008. Characterization and phylogeny of two $\beta$-cytoskeletal actins from Hemibarbus mylodon (Cyprinidae, Cypriniformes), a threatened fish species in Korea. DNA Seq 19, 87-97.

Kim Y, Kim PK, Kim HJ, Jo JY, Han W and Park J. 2009. Effects of water temperature and ambient ammonia concentration on oxygen consumption and ammonia excretion of greenling Hexagrammos otakii Jordan et Starks. Korean J Fish Aquat Sci 42, 373-379.

Kubista M, Andrade JM, Bengtsson M, Forootan A, Jonák J, Lind K, Sindelka R, Sjöback R, Sjögreen B, Strömbom L, Ståhlberg A and Zoric N. 2006. The real-time polymerase chain reaction. Mol Aspects Med 27, 95-125.

Kustermans G, Piette J and Legrand-Poels S. 2008. Actin-targeting nat- 
ural compounds as tools to study the role of actin cytoskeleton in signal transduction. Biochem Pharmacol 76, 1310-1322.

Lee SY, Kim KH and Nam YK. 2009. Molecular characterization of rockbream (Oplegnathus fasciatus) cytoskeletal $\beta$-actin gene and its 5'-upstream regulatory region. Fish Aquat Sci 12, 90-97.

Liu ZJ and Cordes JF. 2004. DNA marker technologies and their applications in aquaculture genetics. Aquaculture 238, 1-37.

Liu ZJ, Moav B, Faras AJ, Guise KS, Kapuscinski AR and Hackett P. 1991. Importance of the CArG box in regulation of $\beta$-actinencoding genes. Gene 108, 211-217.

Miwa T, Manabe Y, Kurokawa K, Kamada S, Kanda N, Bruns G, Ueyama H and Kakunaga T. 1991. Structure, chromosome location, and expression of the human smooth muscle (enteric type) $\gamma$-actin gene: evolution of six human actin genes. Mol Cell Biol 11, 3296-3306.

Nam YK and Kim DS. 2002. Molecular cloning and alternative splicing of growth hormone transcripts in greenling, Hexagrammos otakii. J Korean Fish Soc 35, 676-681.

Nam YK, Maclean N, Hwang G and Kim DS. 2008. Autotransgenic and allotransgenic manipulation of growth traits in fish for aquaculture: a review. J Fish Biol 72, 1-26.

Noh JK, Cho KN, Han EH, Kim A, Lee JS, Kim DS and Kim CG. 2003. Genomic cloning of mud loach Misgurnus mizolepis (Cyprini- formes, Cobitidae) $\beta$-actin gene and usefulness of its promoter region for fish transgenesis. Mar Biotechnol 5, 244-252.

Percipalle P and Visa N. 2006. Molecular functions of nuclear actin in transcription. J Cell Biol 172, 967-971.

Reece KS, McElroy D and Wu R. 1992. Function and evolution of actins. Evol Biol 26, 1-34.

Reisler E and Egelman EH. 2007. Actin structure and function: what we still do not understand. J Biol Chem 282, 36133-36137.

Schmittgen TD and Livak KJ. 2008. Analyzing real-time PCR data by the comparative $\mathrm{C}(\mathrm{T})$ method. Nat Protoc 3, 1101-1108.

Small BC, Murdock CA, Bilodeau-Bourgeois AL, Peterson BC and Waldbieser GC. 2008. Stability of reference genes for real-time PCR analyses in channel catfish (Ictalurus punctatus) tissues under varying physiological conditions. Comp Biochem Physiol B Biochem Mol Biol 151, 296-304.

Truksa J, Lee P and Beutler E. 2007. The role of STAT, AP-1, E-box and TIEG motifs in the regulation of hepcidin by IL-6 and BMP-9: lessons from human HAMP and murine Hamp1 and Hamp2 gene promoters. Blood Cells Mol Dis 39, 255-262.

Venkatesh B, Tay BH, Elgar G and Brenner S. 1996. Isolation, characterization and evolution of nine pufferfish (Fugu rubripes) actin genes. J Mol Biol 259, 655-665. 